\title{
ANISOTROPIC MAGNETIC SUSCEPTIBILITY OF NEODYMIUM SUBSTITUTED $\mathrm{SrLaAlO}_{4} \mathrm{CRYSTALS}^{*}$
}

\author{
J. FinK-FinoWICKI ${ }^{a}$, R. PUŹNIAK ${ }^{a}$, M. BARAN ${ }^{a}$, R. JABLOŃSKI ${ }^{b}$, \\ M. Berkowski ${ }^{a}$, A. PAJĄCZKoWSKa ${ }^{a, b}$ AND H. SzYMCZAK ${ }^{a}$ \\ ${ }^{a}$ Institute of Physics, Polish Academy of Sciences \\ Al. Lotników 32/46, 02-668 Warszawa, Poland \\ ${ }^{b}$ Institute of Electronic Materials Technology \\ Wólczyńska 133, 01-919 Warszawa, Poland
}

(Received October 1, 1993)

\begin{abstract}
The uniaxial anisotropy of magnetic properties of $\mathrm{SrLa}_{1-x} \mathrm{Nd}_{x} \mathrm{AlO}_{4}$ single crystals $(x=0.01$ and 0.05 ) was found from the measurements of temperature dependencies of magnetic susceptibility. Results of measurements, with magnetic field along $a$ - and $c$-axis, are compared with the similar data obtained for $\mathrm{CaNdAlO}_{4}$ crystal. The successful description of experimental data was done in frames of the crystal field approximation. The anisotropy of magnetic susceptibility appears due to crystal field acting on magnetic neodymium ions in a system without exchange interactions.
\end{abstract}

PACS numbers: $75.30 . \mathrm{Hx}, 75.10 . \mathrm{Dg}, 71.70 . \mathrm{Ch}$

\section{Introduction}

Single crystals of neodymium substituted $\mathrm{SrLaAlO}_{4}$ may be of interest as a laser active material. $\mathrm{SrLaAlO}_{4}$ belongs to the group of perovskite-like crystals with $\mathrm{K}_{2} \mathrm{NiF}_{4}$ structure. The crystal is built up, along the $c$ direction, as a sequence of layers containing $\mathrm{AlO}_{6}$ octahedra separated by layers consisting of $\mathrm{Sr}^{2+}$ and $\mathrm{La}^{3+}$ ions more or less randomly distributed in nine-coordinated sites of $C_{4 v}$ symmetry. In investigated crystals a part of La ions are substituted by neodymium ions.

The strong uniaxial anisotropy of magnetic susceptibility has been found in CaNd $\mathrm{AlO}_{4}$ crystal with the same structure $[1,2]$. This anisotropy is introduced by $\mathrm{Nd}^{3+}$ ions. As it was shown by Fink-Finowicki et al. [2], it can appear due to

*This paper was presented at the European Conference "Physics of Magnetism 93", Poznan (Poland), 1993. 
anisotropic exchange interactions between neodymium ions or due to low symmetry crystal field acting on the neodymium ions. Therefore, studies of magnetic properties of neodymium substituted crystals with the same crystallographic structure are very useful to understand deeper the nature of magnetic properties in this class of materials. The aim of this paper is to discuss the origin of magnetic anisotropy of $\mathrm{Nd}$ ions in perovskite-like crystals with $\mathrm{K}_{2} \mathrm{NiF}_{4}$ structure.

\section{Experimental procedure}

Single crystals of $\mathrm{SrLa}_{1-x} \mathrm{Nd}_{x} \mathrm{AlO}_{4}$ (with $x=0.01$ and 0.05 ) and $\mathrm{CaNdAlO}_{4}$ were grown by Czochralski method [3]. Samples with dimensions of few millimeters were X-ray oriented for magnetic measurements performed with magnetic field applied along various crystallographic directions.

Temperature dependence of magnetization of $\mathrm{SrLa}_{0.95} \mathrm{Nd}_{0.05} \mathrm{AlO}_{4}$, as well as $\mathrm{CaNdAlO}_{4}$, has been measured using vibrating sample magnetometer (VSM-PAR Model 155) at temperatures $4.2-160 \mathrm{~K}$ in fixed external magnetic field of $10 \mathrm{kOe}$. The accuracy of magnetic moment measurements was better than $10^{-4} \mathrm{emu}$ and the accuracy of temperature control better than $0.2 \mathrm{~K}$. In the case of $\mathrm{SrLa}_{0.99} \mathrm{Nd}_{0.01} \mathrm{AlO}_{4}$, because of lower contents of magnetic neodymium ions, the magnetization measurements were performed with Quantum Design SQUID magnetometer with resolution of about $10^{-7} \mathrm{emu}$.

\section{Results and discussion}

Magnetization measurements of $\mathrm{SrLa}_{1-x} \mathrm{Nd}_{x} \mathrm{AlO}_{4}$ in the $(a-b)$ plane indicate that susceptibility is isotropic in this plane, similarly as it was observed for $\mathrm{CaNdAlO}_{4}$ [2]. Therefore, the temperature measurements were done for two external magnetic field orientations: along the $a$ - and $c$-axis. The results of these measurements show the uniaxial anisotropy of magnetic susceptibility. The similar result was earlier obtained for $\mathrm{CaNdAlO}_{4}[2]$.

Temperature dependencies of magnetic susceptibility $\chi$ multiplied by temperature $T$ for $\mathrm{CaNdAlO}_{4}$ are presented in wide temperature range up to $760 \mathrm{~K}$ in Fig. 1. The product of $\chi T$ was used in order to get better presentation of data at low temperatures. Anisotropic susceptibility of paramagnetic ions can be described in the frames of the crystal field theory, as it was done e.g. by Elliott and Stevens [4]. In this formalism magnetic susceptibility is given by

$$
\chi=\frac{N \mu_{\mathrm{B}}^{2}}{4 k_{\mathrm{B}} T} \frac{g_{0}^{2}+\sum_{i} g_{i}^{2} \exp \left(-\Delta_{i} / k_{\mathrm{B}} T\right)}{1+\sum_{i} \exp \left(-\Delta_{i} / k_{\mathrm{B}} T\right)}+\left(\chi_{\mathrm{vv}}+\chi_{\mathrm{dia}}\right)
$$

where $g_{0}$ and $g_{i}$ denote $g$-factors and $\Delta_{i}$ are crystal field energy levels. In the above formula terms $\chi_{\mathrm{VV}}$ and $\chi_{\mathrm{dia}}$ represent temperature independent Van-Vleck and diamagnetic susceptibilities. The values of $\Delta_{i}$ for the lowest levels of ${ }^{4} I_{9 / 2}$ ground state of $\mathrm{Nd}^{3+}$ ions in $\mathrm{CaNdAlO}_{4}$ are known [5]. One can expect the anisotropy of $g_{i}$-factors, because of the low symmetry crystal field acting on $\mathrm{Nd}$ ion surrounded by 9 oxygen ions. Fitting the formula (1) to the experimental data, good agreement (see Fig. 1) was obtained for $g_{i}$ values listed in Table and 


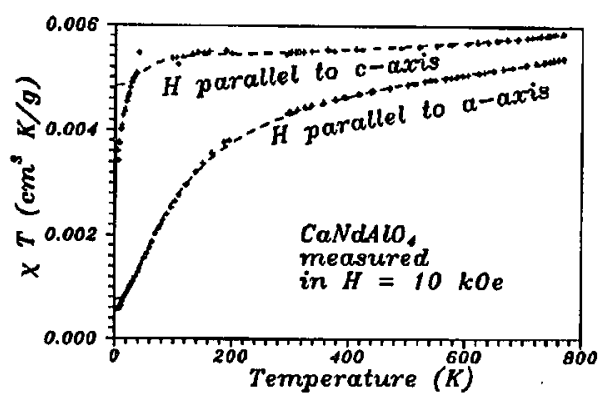

Fig. 1. Temperature dependence of magnetic susceptibility $\chi$ multiplied by temperature $T$ for $\mathrm{CaNdAlO}_{4}$; points (+) - experimental data; dashed lines - crystal field approximation.

$\left(\chi_{\mathrm{VV}}+\chi_{\text {dia }}\right)=10^{-6} \mathrm{~cm}^{3} / \mathrm{g}$. The $g$ values for the lowest energy level are close to values of $g_{0}$ reported from ESR measurements [6] for $\mathrm{SrLaAlO}_{4}$ doped with $5 \%$ of $\mathrm{Nd}$, being equal to 1.55 and 3.67 along $a$ - and $c$-axis, respectively. The values of $g$-factors fitted for higher energy levels are estimated with lower accuracy.

TABLE

The fitted values of $g_{i}$-factors along $a$ - and $c$-axis for $\Delta_{i}$ energy levels of $\mathrm{CaNdAlO}_{4}$ crystals [2]

\begin{tabular}{c|c|c|c|c}
\hline \hline$\Delta_{i} / k_{\mathrm{B}}[\mathrm{K}]$ & 0 & 85.5 & 163 & 289 \\
$g_{i} \| a$-axis & 1.50 & 3.93 & 3.39 & 5.65 \\
$g_{i} \| c$-axis & 3.77 & 4.31 & 4.12 & 2.90
\end{tabular}

On the other hand, the observed dependence of susceptibility is similar to the susceptibility of isolated clusters of interacting magnetic ions (see for example paper by Smart [7]). The successful approximation describing the susceptibility along $a$-axis in the above exchange interaction model requires the assumption of the existence of various neodymium clusters (e.g. pairs and triplets of coupled ions in $a-b$ plane) [2]. It can be realized in $\mathrm{CaNdAlO}_{4}$ lattice with $\mathrm{Ca}^{2+}$ and $\mathrm{Nd}^{3+}$ ions placed randomly in crystallographically equivalent positions.

Experimental results of susceptibility of $\mathrm{CaNdAlO}_{4}$ crystal can be described in crystal field approximation as well as by anisotropy of exchange interactions, and it is impossible to distinguish which approximation better describes experimental data. However, measurements of isostructural crystals with a part of neodymium ions substituted by diamagnetic ions could verify which model is valid. In frames of crystal field approximation the temperature behavior of susceptibility of non-interacting $\mathrm{Nd}$ ions should be similar for various concentrations, while in the anisotropic exchange description this behavior should be strongly dependent on neodymium concentration.

Magnetic susceptibility measured for $\mathrm{SrLa}_{1-x} \mathrm{Nd}_{x} \mathrm{AlO}_{4}$ single crystals, be- 
sides temperature dependent $\chi_{\mathrm{Nd}}$ and temperature independent paramagnetic $\chi_{\mathrm{VV}}$ terms, contains diamagnetic contribution of host crystal $\chi_{\text {dia }}$, which becomes significant at high temperature region, especially for samples with low $\mathrm{Nd}$ concentration. Diamagnetic term has been estimated as $\chi_{\mathrm{dia}}=-1.7 \times 10^{-7} \mathrm{~cm}^{3} / \mathrm{g}$.

Temperature dependent $\chi_{\mathrm{Nd}}$ susceptibility was calculated by subtracting the temperature independent contribution from the measured susceptibility. Final re-

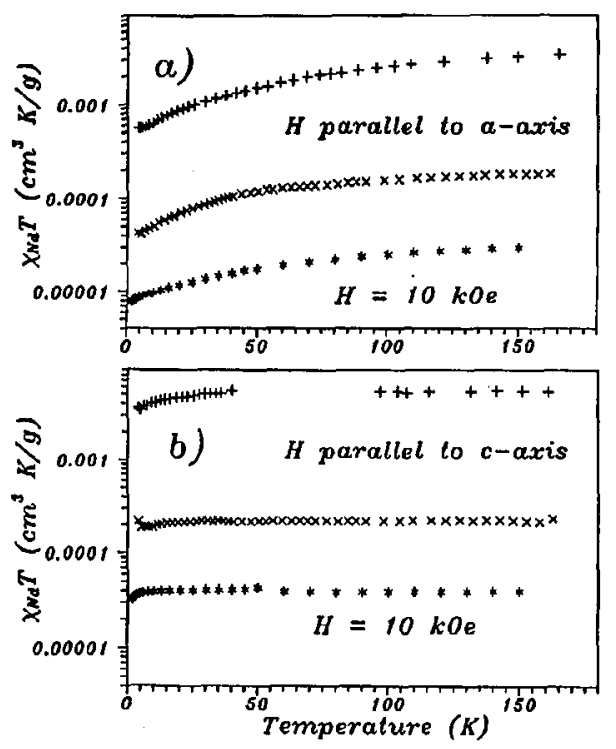

Fig. 2. Temperature dependent $\mathrm{Nd}^{3+}$ contribution to the total susceptibility presented in a logarithmic plot of $\chi_{\mathrm{Nd}} T$ vs. $T$ (a) along $a$-axis and (b) along $c$-axis. Experimental points: $* * * * * 1 \% \mathrm{Nd}, \times \times \times \times \times 5 \% \mathrm{Nd}-$ substituted into $\mathrm{SrLaAlO}_{4}$, and +++++ $\mathrm{CaNdAlO}_{4}$ crystal.

sults of $\chi_{\mathrm{Nd}}$ along $a$-axis and along $c$-axis are presented in Fig. 2a and 2b, respectively, where the values of $\chi_{\mathrm{Nd}} T$ are plotted in logarithmic scale vs. $T$. The lowest curves corresponding to $1 \% \mathrm{Nd}$, and the middle curves corresponding to $5 \%$ of $\mathrm{Nd}$ concentration in $\mathrm{SrLaAlO}_{4}$ are compared with the highest curves for $\mathrm{CaNdAlO}_{4}$. These plots show qualitatively the same temperature behavior for various $\mathrm{Nd}$ contents. Little differences can be due to: (1) experimental errors, especinlly for the lowest $\mathrm{Nd}$ concentration, (2) errors in estimated values of diamagnetic term $\chi_{\text {dia }},(3)$ possible differences between energy levels and $g$-factors for $\mathrm{Nd}^{3+}$ ions in $\mathrm{SrLaAlO}_{4}$ and in $\mathrm{CaNdAlO}_{4}$.

It proves that the origin of observed anisotropic magnetic properties is the same, both in $\mathrm{SrLa}_{1-x} \mathrm{Nd}_{x} \mathrm{AlO}_{4}$ and $\mathrm{CaNdAlO}_{4}$ crystals. Finally, comparison of results obtained for the samples with different concentration of neodymium confirms that observed anisotropic effect, connected with magnetic behavior of $\mathrm{Nd}^{3+}$ ions, is a consequence of anisotropy of neodymium $g$-factor due to low symmetry crystal field. It indicates that the magnetic anisotropy even in the dense paramagnet 
$\mathrm{CaNdAlO}_{4}$ is also due to the same origin.

\section{Acknowledgments}

This work was supported in part by the Committee for Scientific Research under grants No. 8.8035.93.03 and No. 3.3641.93.03.

\section{References}

[1] R. Jabłoński, P. Byszewski, W. Giersz, A. Pajączkowska, I. Pracka, Mater. Lett. 14, 183 (1992).

[2] J. Fink-Finowicki, R. Puźniak, M. Baran, P. Byszewski, M. Gutowski, H. Szymczak, A. Pajaczkowska, presented at EMMA'93, Košice, Slovakia 1993, to be published in IEEE Trans. Magn.

[3] P. Byszewski, A. Pajączkowska, J. Sass, K. Mazur, in: Crystal Properties and Preparation, Vol. 36-38, Ed. A. Lörinczy, Trans. Tech. Publications Ltd., Zürich 1991, p. 560 .

[4] R.J. Elliott, K.W.H. Stevens, Proc. Phys. Soc. A 219, 387 (1953).

[5] W. Ryba-Romanowski, S. Gołąb, J. Hanuza, M. Mączka, A. Pietraszko, M. Berkowski, A. Pajączkowska, J. Phys. Chem. Solids 52, 1043 (1991).

[6] R. Jabłoński, I. Pracka, A. Pajączkowska, in: Abstracts of XV Conference on Radio and Microwave Spectroscopy RAMIS '93, Poznań, 1993, OWN, Poznań 1993, p. 80.

[7] J.S. Smart, in: Magnetism, Vol. III, Eds. G. Rado, H. Suhl, Academic Press, New York 1963, p. 63. 